Wong, A., Boshuizen, H.C., Schellevis, F.G., Kommer, G.J., Polder, J.J. Longitudinal administrative data can b8 used to examine multimorbidity, provided false discoveries are controlled for. Journal of Clinical Epidemiology: 2011, 64(10), 1109-1117

\begin{tabular}{|l|l|}
\hline $\begin{array}{l}\text { Postprint } \\
\text { Version }\end{array}$ & 1.0 \\
\hline Journal website & http://dx.doi.org/10.1016/j.jclinepi.2010.12.011 \\
\hline Pubmed link & http://www.ncbi.nlm.nih.gov/pubmed/21454049 \\
\hline DOI & $10.1016 /$ j.jclinepi.2010.12.011 \\
\hline
\end{tabular}

This is a NIVEL certified Post Print, more info at http://www.nivel.eu

\title{
Longitudinal administrative data can be used to examine multimorbidity, provided false discoveries are controlled for
}

\author{
ALBERT WONG ${ }^{\text {A, B }}{ }^{\text {, HENDRIEK C. BOSHUIZEN }}{ }^{\mathrm{A}}$, FRANÇOIS G. SCHELLEVIS ${ }^{\mathrm{C}, \mathrm{D}}$, GEERT JAN KOMMERE AND \\ JOHAN J. POLDER ${ }^{\mathrm{B}, \mathrm{E}}$
}

${ }^{a}$ Department of Statistics and Mathematical Modeling, Centre for Methodology and Information Services, National Institute for Public Health and the Environment, 3720 BA Bilthoven, The Netherlands

${ }^{b}$ Faculty of Social and Behavioral Sciences, Department of Tranzo, University of Tilburg, 5037 AB Tilburg, The Netherlands

${ }^{\mathrm{c}}$ Department of General Practice, Netherlands Institute for Health Services Research, 3513 CR Utrecht, The Netherlands

${ }^{\mathrm{d}}$ Department of General Practice/EMGO Institute, VU University Medical Centre, 1081 BT Amsterdam, The Netherlands

${ }^{\mathrm{e}}$ Centre for Public Health Forecasting, National Institute for Public Health and the Environment, 3720 BA Bilthoven, The Netherlands

\begin{abstract}
Objective

This article presents methods for using administrative data to study multimorbidity in hospitalized individuals and indicates how the findings can be used to gain a deeper understanding of hospital multimorbidity.

Study Design and Setting

A Dutch nationwide hospital register $(n=4,521,856)$ was used to calculate age- and sexstandardized observed/expected ratios of disease-pairing prevalences with corresponding confidence intervals.

Results

The strongest association was found for the combination between alcoholic liver and mental disorders due to alcohol abuse (observed/expected $=39.2$ ). Septicemia was found to cluster most frequently with other diseases. The consistency of the ratios over time depended on the number of observed cases. Furthermore, the ratios also depend on the length of the time frame considered.
\end{abstract}

\section{Conclusion}

Using observed/expected ratios calculated from the administrative data set, we were able to (1) better quantify known morbidity pairings while also revealing hitherto unnoticed associations, (2) find out which pairings cluster most strongly, and (3) gain insight into which diseases cluster frequently with other diseases. Caveats with this method are finding spurious 
Wong, A., Boshuizen, H.C., Schellevis, F.G., Kommer, G.J., Polder, J.J. Longitudinal administrative data can be used to examine multimorbidity, provided false discoveries are controlled for. Journal of Clinical Epidemiology: 2011

associations on the basis of too few observed cases and the dependency of the ratio magnitude on the length of the time frame observed.

\section{INTRODUCTION}

What is new?

- This article deals with various ways of using observed/expected ratios of prevalences of disease pairs, derived from administrative data, to examine multimorbidity.

- Not only do these ratios reveal associations between diseases that were previously unnoticed but also give insight into which diseases are most strongly associated statistically and which diseases cluster frequently with other diseases.

- For protection against false positive findings, we propose a method that requires consistency of findings over time. Alternatively, as a general rule, we observed that ratios greater than 2 and less than 0.5 , and with observed numbers at least 100 , were found to be consistent.

- Furthermore, researchers should realize that findings depend on the length of time over which the ratios were calculated. As the time frame is expanded, diseases generally tend to cluster stronger.

The increasing occurrence of multimorbidity - the simultaneous presence of more than one disease in an individual - poses several difficulties for society. Not only can multimorbidity be an obstacle for patients in leading a normal and productive life, it also alters the quantity and type of social, medical, and health care services that are needed to support them. Studies[1] $]^{1}$ have shown that multimorbidity is strongly associated with a higher mortality risk [2], [3], [4], [5] and [6], and in many cases, multimorbidity is associated with more health care utilization [7], [8], [9], [10] and [11]. Specific disease combinations have been found to affect functional status and quality of life [12], [13], [14] and [15].

The importance of knowledge in this area is widely acknowledged. Although multimorbidity is often merely used as an explanatory variable in research to adjust for outcomes, the view that it is an object of study is increasingly common. Most studies that focus on multimorbidity fall into one of the following categories. The first type deals with identifying and investigating the clinical relevance of specific disease combinations, usually an index disease and other diseases [16] and [17]. The second focuses on describing the epidemiology of disease combinations that are found among patients, especially in the general population [18] and [19].

Although in its approach it is purely descriptive, this study aims to contribute to the first category. In this article, we examine multimorbidity in the Dutch hospitalized population using data on hospital admissions over a 10-year period from a nationwide Dutch hospital register. Our primary goal is to show how a national hospital register can be used to gain insight into hospital multimorbidity, by examining the associations between all possible disease pairs. We also show how these associations can be calculated, summarized, and presented to form a comprehensive overview of hospital multimorbidity and what pitfalls an analyst may encounter when working with these results. In particular, the role of pure chance has to be addressed. Multiple testing could lead to many accidental associations. Many approaches have been suggested to deal with this. The Benjamini-Hochberg [20] procedure is generally considered as one of the most powerful, but it still can be very conservative. Based on our findings, we propose an alternative way to filter out significant associations that likely follow from chance.

\section{METHODS}

\subsection{Data}

For this study, the Dutch Hospital Discharge Register (LMR [21]) was used. Data are available for the period 1995-2004. All general and academic hospitals and most specialized hospitals in the Netherlands participated in the register during this period. This resulted in a data set that captures nearly all hospital admissions in the Netherlands throughout that period. For each admission, demographic information (date of birth, sex, and postal code) and details regarding the admission (date of admission and discharge, principal and secondary diagnoses, and discharge destination) were recorded. The LMR database was linked to the national Population Register (GBA) by Statistics Netherlands using the date of birth, sex, and postal code that were registered at the time of admission. The linkage allowed for longitudinal analysis of 
Wong, A., Boshuizen, H.C., Schellevis, F.G., Kommer, G.J., Polder, J.J. Longitudinal administrative data can be used to examine multimorbidity, provided false discoveries are controlled for. Journal of Clinical Epidemiology: 2011

hospital care utilization on an individual level and also for obtaining additional patient information, such as the date of decease. Although the aforementioned linkage keys did not allow for a complete identification of individuals, most admissions were successfully linked to individuals (more than $87 \%$ of all admissions [22]). The demographic composition of the resulting data set may differ slightly from that of the Dutch population (in particular, elderly from nonwestern origins more often have a date of birth, that is, not registered correctly and thus are relatively underrepresented) but is nonetheless considered sufficiently representative by Statistics Netherlands.

Diagnoses were recorded in International Classification of Diseases, Ninth Revision (ICD-9) format. They were only recorded by the specialist when relevant to the specific admission in question (i.e., either related to the reason of admission or affecting the length of stay).

\subsection{Definition of multimorbidity}

Multimorbidity is defined here as the co-occurrence of two or more diseases (discharge diagnoses) within one person, in a specific period of time. This is in line with the common definition [23]. For our classification of diseases, we used the International Shortlist for Hospital Morbidity Tabulation (ISHMT) format, which is compiled by the Hospital Data Project group and adopted by Eurostat, Organisation for Economic Co-Operation and Development (OECD), and the World Health Organisation-Family of INternational Classifications (WHO-FIC) network. The ISHMT format features a higher aggregation level than ICD-9 and International Classification of Diseases, Tenth Revision (ICD-10). It uses 138 groups of diseases in total, eight of which are related to external causes (which can never be the principal diagnosis of an admission). ICD-9 codes in our database were converted to ISHMT format using the official ICD to ISHMT conversion table [24]. For our purposes, we considered the ICD-9 format too detailed, as it would feature many theoretical disease pairings that rarely occur in practice. The ISHMT format is developed with the hospital setting in mind. The disease grouping in the ISHMT format includes both chronic and nonchronic nature diseases. For the analysis, we used different time periods: 1, 3, 5, or 10 years. No distinction between principal and secondary diagnoses is made.

\subsection{Population}

The data set contained hospital discharges from the period 1995-2004. To study multimorbidity over different periods of time, we selected several time intervals. For a 1-year period, we took 2004 as the baseline year. Findings from admissions in 1998 and 2001 were compared with 2004, to test for temporal consistency of the results, and also as means of validation. On top of that, 3-year periods (1996-1998, 1999-2001, and the baseline period 2002-2004), 5-year periods (1995-1999 and the baseline period 20002004), and a 10-year period (1995-2004) are selected to examine the effects of scaling of the observation period. The restriction was imposed that patients were uniquely identifiable throughout the whole period and that they were alive during the whole of the particular period. This was done to obtain a more homogenous population with similar person-at-risk times, such that the selected patients had equal "opportunity" to consume care.

\subsection{Disease-tracking level}

Multimorbidity was analyzed on a patient level. Disease combinations were restricted to pairs of diseases. This means that for a patient with three diseases (A, B, and C) within the observed period, three pair combinations were found and counted (AB, AC, and $\mathrm{BC})$. Because the ISHMT format uses 130 diagnosis groups (excluding external causes; see above), counts were tracked for 8,385 combinations in total. In this article, we mainly show combinations of diseases that are of relevance for the elderly, and we excluded some classifications that do not refer to specific diseases, such as admissions related to pregnancy (chapter 15), conditions originating in the perinatal period (chapter 16), congenital malformations (chapter 17), symptoms (chapter 18), injury (chapter 19), and contacts with health services (chapter 21).

\subsection{Observed/expected ratio}

Considering absolute number of persons with a certain disease combination has limited value. The absolute numbers are to a large extent determined by the prevalence rates of each disease in the combination. For instance, heart diseases are part of the most common disease combinations, as the prevalence of these diseases is very high. Therefore, it is more informative to view these prevalences in perspective and compare observed with expected numbers. The latter are the counts that are calculated 
Wong, A., Boshuizen, H.C., Schellevis, F.G., Kommer, G.J., Polder, J.J. Longitudinal administrative data can be used to examine multimorbidity, provided false discoveries are controlled for. Journal of Clinical Epidemiology: 2011

under the assumption of statistical independence of the occurrence of the respective diseases and are found by multiplying the population size with the prevalence rates [19].

These probabilities vary according to the composition of the study population, depending in particular, on age. In order to control for age and sex, a stratified approach was used. Four age groups, further divided according to sex, were chosen with the middle of the observation period as reference $(0-24,25-44,45-64$, and $65+$ ). Thus, the study population was divided into eight strata, and the probabilities $\mathrm{Pk}$ in each stratum $\mathrm{k}$ were weighted with the size Nk of the stratum. The standardized observed/expected ratio is then as follows:

\section{[FIGURE 1]}

where $n k(A)$, nk (B), and nk (AB) denote the number of patients with disease A, B, and both (i.e., the pair $\mathrm{AB})$, respectively, in stratum $\mathrm{k}$.

A ratio greater than 1 implies a positive association, and a ratio smaller than 1 implies a negative association between the two diseases. To assess the statistical significance of these ratios, $95 \%$ lower and upper confidence limits were calculated. The observed counts were assumed to be Poisson distributed, and the Poisson confidence limits were calculated by using Byar's approximations [25].

\subsection{Summarizing the ratios}

The ratios were not only used to confirm known disease pairings and find new ones. In this article, we show listings of which diseases cluster most strongly and which diseases cluster most frequently with other diseases.

\subsection{Validation of the ratios}

The data set spans a number of years. We make use of this aspect by checking consistency of ratios over time. Furthermore, the longitudinal aspect of the data set allowed us to count disease pairs in individuals over different lengths of time. In most studies, a 1-year observation period is taken [18] and [19] often because researchers are interested in short-term clustering or otherwise because of a lack of long-term data. However, the observed degree of disease clustering within individuals might depend on the observation period. We therefore experimented with different lengths of observation periods to see whether certain clusters are only observed over longer periods of time, or whether the time period has little effect on the clustering.

\section{RESULTS}

Characteristics of the hospital study population are found in Table 1, for a few different periods of time. Also shown in Table 1 is the number of primary diagnoses per individual. These numbers are similar over 1-, 3-, and 5-year periods, respectively. As the observation period is longer, the average number of diagnoses per patient increases, and a shift in the distribution is clearly visible. Over 10 years, however, $37 \%$ of the patients still had only one diagnosis.

\section{[TABLE 1]}

In Table 2, the 15 disease pairs most frequently occurring in 2004 are shown. Most pairs involve diabetes mellitus, heart diseases (hypertensive diseases, heart failure, and angina pectoris), and respiratory diseases (chronic obstructive pulmonary disease [COPD] and pneumonia). Some differences may be observed when comparing the 1-year periods, even when adjusted for age and sex (shown between parentheses). The combination diabetes mellitus with heart failure, and combinations of COPD with heart failure, conduction disorders, and diabetes mellitus occurred less often in 2004 than in previous 1-year periods. Differences between periods with similar lengths were found to be smaller when longer observation periods were chosen as basis of measurement (not depicted here). Conversely, diabetes mellitus with hypertensive diseases and cataract with conduction disorders occurred more often in 2004. These combinations are highly prevalent because of high occurrence rates of each separate disease. Note that the percentages 
Wong, A., Boshuizen, H.C., Schellevis, F.G., Kommer, G.J., Polder, J.J. Longitudinal administrative data can be used to examine multimorbidity, provided false discoveries are controlled for. Journal of Clinical Epidemiology: 2011

increase as the observation period increases. This shows, as is to be expected, that pairs of diseases become more prevalent over a longer time period.

\section{[TABLE 2]}

Age- and sex-standardized observed/expected ratios were calculated for all pairs. Outcomes were initially classified as positively associated (ratio significantly greater than 1 ), negatively associated (significantly smaller than 1), and nonassociated (not significantly different from 1). Table 3 gives the 20 strongest associations found in our data, for the year 2004. The top 20 in 2004 seems to be dominated by pairs that correspond to similar diagnosis or body system where the symptoms appear, such as acute lower respiratory infections and COPD. The top 20 in other years are not an identical match, however. This is caused by observed counts that change over time (not depicted). Some combinations show an upward trend in the observed counts, such as the pairings of cholelithiasis with other diseases of gall bladder (from 1,654 in 1998 to 2,240 in 2004) and pancreas (from 745 in 1998 to 1,030 in 2004).

\section{[TABLE 3]}

These varying ratios raise the question whether such approach using an administrative database is sufficiently reliable over time. We tried to answer that question by verifying all results within 1-, 3-, and 5year periods, respectively. Disease pairs were considered as consistent if they either had ratios significantly greater than 1 , ratios significantly smaller than 1 , or ratios not significantly different from 1 , in all periods of similar lengths examined (most of which are found in Table 4). About $80.2 \%$ of all outcomes were consistent for three 1-year periods (1998, 2001, and 2004). About 19.8\% of those outcomes were found to be discordant in at least one of the years. Furthermore, the impact of observed count size and the magnitude of the ratio on the consistency were assessed. Of the inconsistent pairs in the 1 -year periods, $80.8 \%$ had less than 100 observed pairs in all 3 years. About $71.3 \%$ of these cases in which the number of observations had a ratio smaller than 0.5 or larger than 2 in at least one of the years. It shows that even with a large ratio, results can be very unstable because of a small number of observed cases. For those inconsistent pairs observed at least 100 times, the majority $(97.8 \%)$ had a ratio between 0.5 and 2 in all 3 years. Similar findings can be found for 3- and 5-year periods, with the difference that small observed counts became increasingly rare.

\section{[TABLE 4]}

Among these consistent pairs, combinations can be found that are not obvious at first glance and that may draw attention to medically interesting associations. For instance, the groups "diseases of the esophagus" and "paralytic ileus and intestinal obstruction without hernia" were found to cluster (ratio of 2.34 in 2004 , with a corresponding confidence interval of [1.89, 2.79]). Ileus is a well-known complication of gastrointestinal surgery. It is likely that the combination occurs with the ileus being a complication of surgery for an esophagus disorder, but this invites further research. Other interesting pairings include anaemia and schizophrenia $(2.33[1.99,2.71])$, anemia and dementia $(2.85[2.56,3.16])$, "glomerular and renal tubulointerstitial diseases" and "other diseases of the liver" $(3.64[3.01,4.36])$, and dermatitis and renal failure $(4.60[3.77,5.56])$.

Table 5 shows the 30 diseases with the greatest number of strong associations, where "strong" means a ratio smaller than 0.5 or greater than 2.0, for 1-, 3-, 5-, and 10-year periods. Septicemia, anemia, and renal failure are often found to be strongly associated to other diseases. Other diseases that are of interest are dermatitis, eczema, and diabetes mellitus. These diseases are remarkable in that most associations are positive.

\section{[TABLE 5]}


Wong, A., Boshuizen, H.C., Schellevis, F.G., Kommer, G.J., Polder, J.J. Longitudinal administrative data can be used to examine multimorbidity, provided false discoveries are controlled for. Journal of Clinical Epidemiology: 2011

The length of the observed time period was found to have a substantial effect on the association patterns over which multimorbidity is observed. With longer periods of observation, the numbers of "strong associations" were seen to increase, an increase for the 'strong associations' and the 'remainder' category is visible with a larger time period. With a longer period, it is more likely that the individuals will suffer from more than one disease. Thus, over a longer period, more clustering of diseases is found. This is virtually the case for all combinations. For most ratios, this meant a slight increase in magnitude. Pairs for which a change in outcome was found as the length of the observation period increased include atherosclerosis and dyspepsia (ratio 2004: 1.04 [0.77, 1.37], ratio 1995-2004: 2.19 [2.03, 2.26]), infections of the skin and glomerular diseases $(1.04[0.76,1.40]$ and $2.23[2.06,2.41]$, respectively), and dyspepsia and dorsalgia $(1.13[0.80,1.55]$ and $2.26[2.08,2.45])$. Dyspepsia, dorsalgia, diarrhea, and gastroenteritis are conditions that frequently formed part of these "temporally inconsistent" pairs. Some combinations share a common risk factor (alcoholic liver disease and intracranial injury, $1.08[0.47,2.14]$ and $3.23[2.73,3.78]$ ).

\section{DISCUSSION}

\subsection{Summary of results}

This article sought to give an overview of methods that may be used to study multimorbidity when a hospital administrative data set is available. Using standardized observed/expected ratios, the strengths of the pairwise associations between diseases, allowing the pairs to be classified as positively associated, negatively associated, or not associated. This information can be used to confirm known occurrence relations between diseases or search for hitherto unknown associations. If ratios are estimated for all possible pairings, additional information can be derived. Listings can be made of the pairings with the greatest ratios, that is, the strongest associations. Furthermore, it can be identified which diseases cluster most frequently with other diseases. However, a problem with these ratios is that results become unreliable when observed numbers are smaller than 100 and when ratios are close to 1 . The magnitude of the ratios can also be sensitive to the length of the time interval over which they are estimated.

\subsection{Strengths and weaknesses of this study}

As far as we know, no other study has examined multimorbidity among hospital patients in such detail. Multimorbidity has been examined in a similar fashion in the Dutch general practice population [18] and [19]. Also cluster analysis of US primary care data has been used to assess multimorbidity [26]. Specific hospital multimorbidity has been studied by analyzing statistical associations in UK hospital data [27]. We made calculations for all diseases, classified according to the ISHMT format. But these are easily replicable using other formats such as ICD-9 and ICD-10 if further differentiating is needed. The major strength is the use of a data set that encompasses nearly all Dutch hospital admissions. The scope of this data set allows an accurate description of multimorbidity of hospital patients. A novelty of this study is its approach for analyzing multimorbidity over a long term. The same method of comparing observed with expected frequencies was applied using different time intervals of observation. Although this adds little value from an etiological point of view, it yields new insights into how multimorbidity evolves over time. Also, we performed the analysis for several calendar years to further validate our findings.

However, this data set did not come without some limitations. First, we had to make a choice on how to deal with individuals who were not alive during the whole period (births and deaths). Our choice to exclude these to obtain a more homogenous study population also introduced a source of bias. Particularly those diseases with a high mortality rate (such as the cancers, acute myocardial infarction, and other heart diseases) were slightly underrepresented in this study. However, a sensitivity analysis showed that these selection criteria did not affect the results in a drastic way. The combinations that were sensitive to these criteria were mainly those that had small observed counts in the first place. Second, the validity of this study depends strongly on how accurate diagnoses were recorded in the administrative process. Also, discharge hospital diagnoses were only recorded when they were relevant to the specific admission in question (i.e., they either were related to the reason of admission or affected the length of stay). This would probably lead to underreporting of specific diseases [28]. Furthermore, we cannot estimate the frequency and impact of misdiagnoses. We also observed time trends in some diagnostic pairings, without being able to determine their source. The pairings might truly increase or decrease in frequency over time or might simply be a reflection in improvements in diagnostics or a change in classification of the disease. However, 
Wong, A., Boshuizen, H.C., Schellevis, F.G., Kommer, G.J., Polder, J.J. Longitudinal administrative data can be used to examine multimorbidity, provided false discoveries are controlled for. Journal of Clinical Epidemiology: 2011

no financial incentives are in place for registering specific diagnoses, and it is unlikely the results of register itself have affected the registration procedure over time (e.g., for purposes of quality control). The Dutch Hospital Register as used in this study is a register funded by public money and was set up with the goal of promoting research in medicine and related disciplines. Hospitals did not get compensated for the number of diagnoses as recorded in this register, but they were compensated by global budgets as set by the Dutch government.

Finally, some further potential sources of bias should be mentioned. Thus, the so-called surveillance bias could result if admittance to the hospital for a disease A increases the probability of being diagnosed with disease B. However, if this bias would have been involved, we would expect it to be equally present for all disease combinations. As our data set was restricted to the hospital setting, referral bias certainly also played a role because diseases differ in the likelihood of hospital admission and are treated more exclusively than in other care providers. Just as is the case with surveillance bias, it is difficult to quantify the influence it may have had on our findings.

\subsection{Implications for future research on multimorbidity and health policy}

This study confirms the potential of using large administrative data sets such as the Dutch Hospital Discharge Register for research. Using an automated approach, statistical associations were deducted for each possible pairs of diseases. This approach is an effective way to confirm and further quantify the prevalence of disease combinations that are known in the literature. But above all, it allows us to detect hitherto unknown associations between diseases. Although the nature and clinical significance of these associations cannot be deducted from administrative data, the finding that there is a statistical association is revealing in itself, prompting further research on the relationship of the respective diseases. In addition, an administrative data set allows for a comprehensive listing of the most strongly associated pairings and the most frequently associated diseases. In combination with the absolute numbers, these can give researchers additional clues as to which pairings may be given priority in further research and formulating health care policies.

However, in considering observed/expected ratios estimated in the manner described in this study, researchers should keep the following in mind. Patterns of multimorbidity vary over time. Although our approach was purely statistical, allowing us no insight into the causal mechanisms behind the observed associations, it did become clear that for many diseases a longer period of observations is required before clustering becomes manifest. Thus, clinical and health care researchers interested in assessing etiological relationships should consider this time dependency. The second issue is the variability over time of these ratios. Our analyses show that there is reason for caution when interpreting observed/expected ratios. Although from a strictly statistical point of view, any ratio that differs significantly from 1 can be interpreted as meaning that there is an association, our data show these requirements are not sufficient for practical purposes. The outcomes were sensitive to the observed count sizes and the relative magnitude of the ratio. It reaffirmed the notion that consistency needs to be formally checked for all findings. These caveats are especially relevant in a study as this one, which relies heavily on administrative data, and does not provide any biomedical support for the nature of the associations [29].

One main contribution of this article is that we propose a way to deal with these inconsistent findings, which point toward chance, as an alternative to multiple testing corrections such as Benjamini-Hochberg [20]. Although these corrections are useful when the researcher has no external data to test for validity (which is the case in many studies), they are conservative in terms of the false discovery rate because they are purely based on the probability theory. In our case, we have multiple years of data to check the findings against. It is highly unlikely that a chance finding is consistently reproduced over multiple years (barring any systematic bias because of diagnostic procedures), so information on consistency can be used to filter out chance findings. Suppose the false discovery rate is $\alpha$ in a given year. Assuming independence, the rate of finding a pairing that has seen a significant ratio by chance in $\mathrm{n}$ years is $\alpha$. In our case, the false discovery rate is $0.05^{3}=0.000125$ or 1 of 8,000 . Second, if this is not possible (e.g., because no longitudinal data are available), we propose to use our general rule of thumb of only considering disease pairings with an observed/expected ratio outside the $(0.5,2)$ interval and with an observed count of at least 100.

Information on specific disease pairings derived from administrative databases can be used by health care providers (in this case, hospital managements) to improve the quality of care and optimize the efficiency of 
Wong, A., Boshuizen, H.C., Schellevis, F.G., Kommer, G.J., Polder, J.J. Longitudinal administrative data can be used to examine multimorbidity, provided false discoveries are controlled for. Journal of Clinical Epidemiology: 2011

health care processes. Care should not only be directed at specific diseases but at specific combinations of diseases as well. In particular, the complicating diseases are of great interest to these providers.

Governments should consider the complexity of multimorbidity and acknowledge that improving quality and efficiency of care, and having detailed insight into health care costs, requires a deeper understanding of multimorbidity. To achieve that goal, extensive data sets such as ours are needed.

\section{ACKNOWLEDGMENTS}

This study was funded by VWS, the Dutch Ministry of Health, Welfare and Sport. The authors also thank Agnes de Bruin, Jos de Ree, and Janneke Ploemacher of Statistics Netherlands for providing data and offering helpful comments. Furthermore, the article benefited from insightful comments of Peter Engelfriet and Lany Slobbe of the Dutch National Institute for Public Health and Environment, Dolf de Boer and Sandra van Beek from the Netherlands Institute for Health Services Research.

\section{REFERENCES}

[1] R. Gijsen, N. Hoeymans, F.G. Schellevis, D. Ruwaard, W.A. Satariano and G.A. van den Bos, Causes and consequences of comorbidity: a review, J Clin Epidemiol 54 (2001), pp. 661-674.

[2] M.E. Charlson, P. Pompei, K.L. Ales and C.R. MacKenzie, A new method of classifying prognostic comorbidity in longitudinal studies: development and validation, J Chronic Dis 40 (5) (1987), pp. 373-383. a clinical comorbidity index for use with ICD-9-CM administrative databases, J Clin Epidemiol 45 (1992), pp. 613-619.

[4] P.S. Romano, L.L. Roos and J.G. Jollis, Adapting a clinical comorbidity index for use with ICD-9-CM administrative data: differing perspectives, J Clin Epidemiol 46 (1993), pp. 1075-1079 discussion 108190.

[5] D.A. Southern, H. Quan and W.A. Ghali, Comparison of the Elixhauser and Charlson/Deyo methods of comorbidity measurement in administrative data, Med Care 42 (2004), pp. 355-360.

[6] V. Sundararajan, T. Henderson, C. Perry, A. Muggivan, H. Quan and W.A. Ghali, New ICD-10 version of the Charlson comorbidity index predicted in-hospital mortality, J Clin Epidemiol 57 (2004), pp. 1288-1294.

[7] M. Shwartz, L.I. Iezzoni, M.A. Moskowitz, A.S. Ash and E. Sawitz, The importance of comorbidities in explaining differences in patient costs, Med Care 34 (1996), pp. 767-782.

[8] C.J. Roe, E. Kulinskaya, N. Dodich and W.R. Adam, Comorbidities and prediction of length of hospital stay, Aust N Z J Med 28 (6) (1998), pp. 811-815.

[9] J. Librero, S. Peiro and R. Ordinana, Chronic comorbidity and outcomes of hospital care: length of stay, mortality, and readmission at 30 and 365 days, J Clin Epidemiol 52 (1999), pp. 171-179.

[10] G.P. Westert, W.A. Satariano, F.G. Schellevis and G.A. van den Bos, Patterns of comorbidity and the use of health services in the Dutch population, Eur J Public Health 11 (2001), pp. 365-372.

[11] J.N. Struijs, C.A. Baan, F.G. Schellevis, G.P. Westert and G.A. van den Bos, Comorbidity in patients with diabetes mellitus: impact on medical health care utilization, BMC Health Serv Res 6 (2006), p. 84.

[12] Z. Fuchs, T. Blumstein, I. Novikov, A. Walter-Ginzburg, M. Lyanders and J. Gindin et al., Morbidity, comorbidity, and their association with disability among community-dwelling oldest-old in Israel, J Gerontol A Biol Sci Med Sci 53 (6) (1998), pp. M447-M455.

[13] J. Xuan, L.J. Kirchdoerfer, J.G. Boyer and G.J. Norwood, Effects of comorbidity on health-related quality-of-life scores: an analysis of clinical trial data, Clin Ther 21 (1999), pp. 383-403.

[14] L.P. Fried, K. Bandeen-Roche, J.D. Kasper and J.M. Guralnik, Association of comorbidity with disability in older women: the Women's Health and Aging Study, J Clin Epidemiol 52 (1999), pp. 27-37.

[15] M. Rijken, M. van Kerkhof, J. Dekker and F.G. Schellevis, Comorbidity of chronic diseases: effects of disease pairs on physical and mental functioning, Qual Life Res 14 (2005), pp. 45-55.

[16] J. Nuyen, F.G. Schellevis, W.A. Satariano, P.M. Spreeuwenberg, M.D. Birkner and G.A. van den Bos et al., Comorbidity was associated with neurologic and psychiatric diseases: a general practice-based controlled study, J Clin Epidemiol 59 (2006), pp. 1274-1284.

[17] R. John, D.S. Kerby and C.H. Hennessy, Patterns and impact of comorbidity and multimorbidity among community-resident American Indian elders, Gerontologist 43 (2003), pp. 649-660.

[18] M. van den Akker, F. Buntinx, J.F. Metsemakers, S. Roos and J.A. Knottnerus, Multimorbidity in general practice: prevalence, incidence, and determinants of co-occurring chronic and recurrent diseases, J Clin Epidemiol 51 (1998), pp. 367-375.

[19] M. van den Akker, F. Buntinx, S. Roos and J.A. Knottnerus, Problems in determining occurrence rates of multimorbidity, J Clin Epidemiol 54 (2001), pp. 675-679. 
Wong, A., Boshuizen, H.C., Schellevis, F.G., Kommer, G.J., Polder, J.J. Longitudinal administrative data can b used to examine multimorbidity, provided false discoveries are controlled for. Journal of Clinical Epidemiology: 2011

[20 Y. Benjamini and Y. Hochberg, Controlling the false discovery rate: a practical and powerful approach to multiple testing, J R Stat Soc Series B Stat Method 57 (1) (1995), pp. 289-300.

[21] DHD. Dutch Hospital Data.

[22] A. de Bruin, J.W.P.F. Kardaun, A. Gast, E.I. de Bruin, M. van Sijl and G.C.G. Verweij, Record linkage of hospital discharge register with population register: experiences at Statistics Netherlands, Stat J UN Econ Comm Eur 21 (2004), pp. 23-32.

[23] M. van den Akker, F. Buntinx and J.A. Knottnerus, Comorbidity or multimorbidity: what's in a name? A review of literature, Eur J Gen Pract 2 (1996), pp. 65-70.

[24] WHO. WHO International Shortlist of Hospital Morbidity Tabulation.

[25] N.E. Breslow and N.E. Day, Statistical methods in cancer research. Volume II-the design and analysis of cohort studies, IARC Sci Publ (82) (1987), pp. 1-406.

[26] J.E. Cornell, J.A. Pugh, J.W. Williams Jr., L. Kazis, A.F.S. Lee and M.L. Parchman et al., Multimorbidity clusters: clustering binary data from multimorbidity clusters: clustering binary data from a large administrative medical database, Appl Multivar Res 12 (3) (2008), pp. 163-182.

[27] M. Goldacre, L. Kurina, D. Yeates, V. Seagroatt and L. Gill, Use of large medical databases to study associations between diseases, QJM 93 (2000), pp. 669-675.

[28] M.T. Schram, D. Frijters, E.H. van de Lisdonk, J. Ploemacher, A.J. de Craen and M.W. de Waal et al., Setting and registry characteristics affect the prevalence and nature of multimorbidity in the elderly, $\mathrm{J}$ Clin Epidemiol 61 (2008), pp. 1104-1112.

[29] G. Taubes, Epidemiology faces its limits, Science 269 (1995), pp. 164-169.

\section{[TABLES AND FIGURES]}

\section{[FIGURE 1]}

$$
\begin{aligned}
\text { Ratio } & =\frac{\sum_{k} P_{k}(A B) N_{k}}{\sum_{k} P_{k}(A) P_{k}(B) N_{k}}=\frac{\sum_{k} \frac{n_{k}(A B)}{N_{k}} N_{k}}{\sum_{k} \frac{n_{k}(A)}{N_{k}} \frac{n_{k}(B)}{N_{k}} N_{k}} \\
& =\frac{n(A B)}{\sum_{k} \frac{n_{k}(A) n_{k}(B)}{N_{k}}}
\end{aligned}
$$


Wong, A., Boshuizen, H.C., Schellevis, F.G., Kommer, G.J., Polder, J.J. Longitudinal administrative data can be used to examine multimorbidity, provided false discoveries are controlled for. Journal of Clinical Epidemiology: 2011

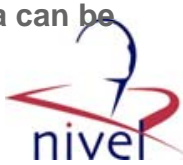

\section{[TABLE 1]}

Table 1

Characteristics of the hospital population, over different observation periods

\begin{tabular}{|c|c|c|c|c|}
\hline Characteristics & $2004(n=1,414,142)$ & $2002-2004(n=2,880,410)$ & $2000-2004(n=4,138,529)$ & $1995-2004(n=4,521,856)$ \\
\hline \multicolumn{5}{|l|}{$\overline{\text { Age/sex }(\%)}$} \\
\hline $0-24$, Male & 9.0 & 9.3 & 10.6 & 9.1 \\
\hline $0-24$, Female & 7.9 & 8.3 & 9.2 & 8.4 \\
\hline $25-44$, Male & 7.6 & 8.3 & 8.9 & 9.8 \\
\hline 25-44, Female & 16.5 & 17.5 & 17.8 & 17.8 \\
\hline 45-64, Male & 13.5 & 13.8 & 13.8 & 15.3 \\
\hline 45-64, Female & 15.0 & 15.0 & 15.2 & 16.6 \\
\hline$\geq 65$, Male & 13.6 & 12.0 & 10.4 & 9.5 \\
\hline$\geq 65$, Female & 17.1 & 15.8 & 14.1 & 13.5 \\
\hline \multicolumn{5}{|c|}{ Diagnoses per individual within period $(\%)$} \\
\hline 1 & 56.7 & 49.1 & 43.0 & 37.0 \\
\hline 2 & 23.6 & 24.5 & 24.8 & 23.3 \\
\hline 3 & 10.4 & 12.5 & 14.0 & 15.0 \\
\hline 4 & 4.4 & 5.9 & 7.3 & 8.9 \\
\hline$\geq 5$ & 5.0 & 8.0 & 10.9 & 15.8 \\
\hline Mean diagnoses & 1.8 & 2.1 & 2.4 & 2.8 \\
\hline
\end{tabular}

Notes: Shares of age/sex and diagnoses per individual strata in the hospital population are given in crude percentages, whereas mean diagnoses are expressed in terms of number of diagnoses per individual. Because of space considerations, numbers are not shown for all studied periods of time.

\section{[TABLE 2]}

Table 2

Crude prevalences of disease pairs and indirectly standardized prevalences (with respect to age and sex distribution in 2004) between parentheses, for different periods of time

\begin{tabular}{|c|c|c|c|c|c|c|}
\hline Group & Group & $\begin{array}{l}1998 \\
(n=1,177,179)\end{array}$ & $\begin{array}{l}2004 \\
(n=1,414,142)\end{array}$ & $\begin{array}{l}2002-2004 \\
(n=2,880,410)\end{array}$ & $\begin{array}{l}2000-2004 \\
(n=4,138,529)\end{array}$ & $\begin{array}{l}1995-2004 \\
(n=4,521,856)\end{array}$ \\
\hline Ear & Tonsils & $1.86(1.56)$ & $1.27(-)$ & $1.65(1.58)$ & $1.98(1.68)$ & $1.66(1.60)$ \\
\hline Diabetes & Hypertensive & $0.34(0.36)$ & $0.40(-)$ & $0.46(0.49)$ & $0.47(0.54)$ & $0.56(0.64)$ \\
\hline Diabetes & $\mathrm{CDO}$ & $0.29(0.31)$ & $0.29(-)$ & $0.35(0.38)$ & $0.37(0.45)$ & $0.44(0.54)$ \\
\hline Diabetes & Heart failure & $0.31(0.33)$ & $0.24(-)$ & $0.28(0.30)$ & $0.28(0.33)$ & $0.31(0.38)$ \\
\hline $\mathrm{HF}$ & COPD & $0.32(0.35)$ & $0.21(-)$ & $0.24(0.26)$ & $0.24(0.29)$ & $0.28(0.35)$ \\
\hline $\mathrm{CDO}$ & COPD & $0.27(0.29)$ & $0.20(-)$ & $0.26(0.29)$ & $0.28(0.34)$ & $0.35(0.44)$ \\
\hline Diabetes & COPD & $0.25(0.27)$ & $0.20(-)$ & $0.21(0.23)$ & $0.21(0.24)$ & $0.23(0.28)$ \\
\hline Anemias & Diabetes & $0.17(0.18)$ & $0.18(-)$ & $0.21(0.23)$ & $0.21(0.25)$ & $0.24(0.28)$ \\
\hline Anemias & Heart failure & $0.18(0.19)$ & $0.17(-)$ & $0.20(0.22)$ & $0.20(0.24)$ & $0.23(0.29)$ \\
\hline Anemias & $\mathrm{CDO}$ & $0.16(0.17)$ & $0.17(-)$ & $0.23(0.25)$ & $0.25(0.30)$ & $0.32(0.40)$ \\
\hline Diabetes & CVD & $0.19(0.21)$ & $0.16(-)$ & $0.21(0.22)$ & $0.22(0.26)$ & $0.27(0.32)$ \\
\hline Diabetes & AP & $0.17(0.19)$ & $0.15(-)$ & $0.21(0.22)$ & $0.26(0.30)$ & $0.36(0.41)$ \\
\hline $\mathrm{CDO}$ & Pneumonia & $0.15(0.16)$ & $0.14(-)$ & $0.20(0.21)$ & $0.21(0.26)$ & $0.27(0.34)$ \\
\hline LM uterus & MMP & $0.19(0.20)$ & $0.14(-)$ & $0.21(0.21)$ & $0.31(0.30)$ & $0.49(0.45)$ \\
\hline Diabetes & Cataract & $0.20(0.21)$ & $0.14(-)$ & $0.31(0.33)$ & $0.43(0.51)$ & $0.61(0.73)$ \\
\hline
\end{tabular}

Abbreviations: $\mathrm{CDO}$, conduction disorder; $\mathrm{HF}$, heart failure; COPD, chronic obstructive pulmonary disease; CVD, cerebrovascular disease; AP, angina pectoris; LM, leiomyoma; MMP, menstrual and menopausal disease.

Listed pairs refer to the 15 most common pairs in 2004.

Notes: "Other" disease groups and pairs within one disease chapter are not shown here. Because of space considerations, numbers are not shown for all studied periods of time. 
Wong, A., Boshuizen, H.C., Schellevis, F.G., Kommer, G.J., Polder, J.J. Longitudinal administrative data can be used to examine multimorbidity, provided false discoveries are controlled for. Journal of Clinical Epidemiology: 2011

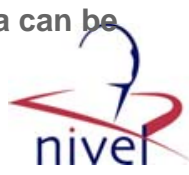

[TABLE 3]

Table 3

Disease pairs with the highest O/E ratio in 2004, compared with two other years (1998 and 2001)

\begin{tabular}{|c|c|c|c|}
\hline \multirow[b]{2}{*}{ Disease 1} & \multirow[b]{2}{*}{ Disease 2} & \multirow{2}{*}{$\frac{1998}{\mathrm{O} / \mathrm{E}(95 \% \mathrm{CI})}$} & \multirow{2}{*}{$\frac{2004}{\mathrm{O} / \mathrm{E}(95 \% \mathrm{CI})}$} \\
\hline & & & \\
\hline Mental disorders (alcohol) & Alcoholic liver disease & $47.9(43.1,53.1)$ & $39.2(35.2,43.5)$ \\
\hline Alcoholic liver disease & Other diseases of liver & $30.2(26.1,34.7)$ & $34.3(30.2,38.8)$ \\
\hline Mood (affective) disorders & Other mental and behavioral & $27.3(26.0,28.6)$ & $27.4(26.0,28.9)$ \\
\hline Other acute lower respiratory infections & COPD & $15.7(15.0,16.4)$ & $22.9(21.8,24.0)$ \\
\hline Diseases of gall bladder & Diseases of pancreas & $21.4(19.1,23.9)$ & $22.4(20.3,24.6)$ \\
\hline HIV & Other infectious and parasitic diseases & $23.1(19.9,26.6)$ & $22.3(19.0,26.0)$ \\
\hline Mental disorders (alcohol) & Mental disorders (psych. subst.) & $20.4(18.5,22.6)$ & $21.5(19.8,23.3)$ \\
\hline Cholelithiasis & Other diseases of gall bladder & $18.7(17.9,19.7)$ & $21.1(20.3,22.0)$ \\
\hline Diseases of esophagus & Dyspepsia & $17.6(16.3,19.0)$ & $19.8(18.5,21.2)$ \\
\hline Cholelithiasis & Diseases of pancreas & $17.1(15.9,18.3)$ & $19.5(18.3,20.7)$ \\
\hline Glomerular/renal tubulointerstitial dis. & Renal failure & $21.9(20.2,23.7)$ & $18.9(17.5,20.3)$ \\
\hline Peptic ulcer & Dyspepsia & $14.1(12.9,15.4)$ & $18.1(16.5,19.8)$ \\
\hline Mental disorders (alcohol) & Diseases of pancreas & $16.7(14.9,18.7)$ & $17.8(16.1,19.7)$ \\
\hline Malignant neoplasm of ovary & Other malignant neoplasms & $15.7(14.5,17.0)$ & $17.5(16.3,18.8)$ \\
\hline Diseases of esophagus & Abdominal hernia & $17.9(17.0,18.9)$ & $17.2(16.3,18.1)$ \\
\hline Infectious and parasitic diseases & Acute lower respiratory infections & $16.0(15.2,16.9)$ & $16.8(15.9,17.8)$ \\
\hline Systemic connective tissue disorders & Glomerular/renal tubulointerstitial dis. & $11.4(9.6,13.4)$ & $15.1(13.1,17.4)$ \\
\hline Glomerular/renal tubulointerstitial dis. & Urolithiasis & $13.7(12.5,14.9)$ & $14.6(13.5,15.7)$ \\
\hline Diseases of esophagus & Peptic ulcer & $10.8(9.7,11.9)$ & $13.9(12.6,15.3)$ \\
\hline HIV & Pneumonia & $12.6(9.6,16.1)$ & $13.7(10.4,17.6)$ \\
\hline
\end{tabular}

Abbreviations: E, expected; O, observed; dis., disease; Psych. subst., psychoactive substance; ISHMT, International Shortlist for Hospital Morbidity Tabulation.

Notes: Selection of disease pairs limited to pairs from different ISHMT disease chapters (excluding the chapters concerning symptoms, injury, external causes, and contacts with health services), with at least 50 observed pairs, shown here. Because of space considerations, numbers are not shown for all studied periods of time. 
Wong, A., Boshuizen, H.C., Schellevis, F.G., Kommer, G.J., Polder, J.J. Longitudinal administrative data can b8 used to examine multimorbidity, provided false discoveries are controlled for. Journal of Clinical Epidemiology: 2011

\section{[TABLE 4]}

\section{Table 4}

Consistency of disease pair associations within an observation period length

\begin{tabular}{lcll}
\hline & Count $(\%)$ & & \\
\cline { 2 - 4 } Type of association & $1 \mathrm{yr}(3$ periods $)$ & $5 \mathrm{yr}(2$ periods $)$ & $10 \mathrm{yr}(1$ period $)$ \\
\hline Total & $8,385(100)$ & $8,385(100)$ & $8,385(100)$ \\
Consistent pairs & $6,723(80.2)$ & $7,310(87.2)$ & $8,385(100)$ \\
Inconsistent pairs & $1,662(19.8)$ & $1,075(12.8)$ & - \\
Consistent pairs & $6,723(100)$ & $7,310(100)$ & $8,385^{\mathrm{a}}(100)$ \\
Total positive & $1,586(23.6)$ & $2,883(39.4)$ & $3,801(45.3)$ \\
associations & & & \\
O/E $\geq 2$ & $866(12.9)$ & $1,338(18.3)$ & $1,653(19.7)$ \\
Remainder & $720(10.7)$ & $1,545(21.1)$ & $2,148(25.6)$ \\
Total negative & $4,250(63.2)$ & $3,618(49.5)$ & $3,284(39.2)$ \\
associations & & & \\
O/E $\leq 0.5$ & $3,310(49.2)$ & $1,331(18.2)$ & $751(9.0)$ \\
Remainder & $940(14.0)$ & $2,287(31.3)$ & $2,533(30.2)$ \\
No associations & $887(13.2)$ & $809(11.1)$ & $1,300(15.5)$ \\
Inconsistent pairs & $1,662(100)$ & $1,075(100)$ & - \\
$\mathrm{O}<100,0.5 \leq \mathrm{O} / \mathrm{E} \leq 2$ & $386(23.2)$ & $202(18.8)$ & - \\
$\mathrm{O}<100$, Remainder & $957(57.6)$ & $293(27.3)$ & - \\
$\mathrm{O} \geq 100,0.5 \leq \mathrm{O} / \mathrm{E} \leq 2$ & $312(18.8)$ & $530(49.3)$ & - \\
$\mathrm{O} \geq 100$, Remainder & $7(0.4)$ & $50(4.7)$ & - \\
\hline
\end{tabular}

Abbreviations: O, observed; E, expected.

Notes: A disease pair is only considered to be consistent when the outcome (association, negative association, or no association) is equal in all periods, for the given observation period length. Likewise, an (dis)association is only defined as strong if the ratio is at least 2 (at most 0.5 ) and statistically significant in all periods. Consistency check could only be done for two 5-year periods, a no check could be made for 10 years. Because of space considerations, numbers are not shown for a period length of 3 years.

a Because no check could be made on the 10-year period, the distribution of consistent and inconsistent pairs was not known. For indication purposes only, all pairs over 10-year period were considered to be consistent and described accordingly in the table. 
Wong, A., Boshuizen, H.C., Schellevis, F.G., Kommer, G.J., Polder, J.J. Longitudinal administrative data can b8 used to examine multimorbidity, provided false discoveries are controlled for. Journal of Clinical Epidemiology: 2011

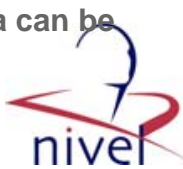

\section{[TABLE 5]}

Table 5

Top 30 diseases with the highest number of strong statistical associations $(\mathrm{O} / \mathrm{E} \geq 2)$ in 1 year, shown together with strong negative associations $(\mathrm{O} / \mathrm{E} \leq 0.5)$ and a remainder category, containing weak (dis)associations and nonassociations

\begin{tabular}{|c|c|c|c|c|c|c|c|c|c|}
\hline \multirow[b]{2}{*}{ Disease } & \multicolumn{3}{|c|}{ 1yr: O/E } & \multicolumn{3}{|c|}{$5 \mathrm{yr}: \mathrm{O} / \mathrm{E}$} & \multicolumn{3}{|c|}{ 10yr: $\mathrm{O} / \mathrm{E}$} \\
\hline & $\geq 2.0$ & $\leq 0.5$ & $R$ & $\geq 2.0$ & $\leq 0.5$ & $R$ & $\geq 2.0$ & $\leq 0.5$ & $R$ \\
\hline Septicemia & 54 & 13 & 62 & 70 & 5 & 54 & 75 & 1 & 53 \\
\hline Anemias & 48 & 12 & 69 & 61 & 3 & 65 & 67 & 1 & 61 \\
\hline Other endocrine, nutritional, and metabolic dis. & 45 & 23 & 61 & 54 & 2 & 73 & 61 & 0 & 68 \\
\hline Other infectious and parasitic dis. & 43 & 13 & 73 & 54 & 2 & 73 & 64 & 0 & 65 \\
\hline Other dis. of liver & 41 & 16 & 72 & 49 & 5 & 75 & 56 & 1 & 72 \\
\hline Other dis. of the blood & 38 & 13 & 78 & 54 & 3 & 72 & 57 & 1 & 71 \\
\hline Renal failure & 37 & 20 & 72 & 60 & 10 & 59 & 66 & 11 & 52 \\
\hline Other dis. of the urinary system & 37 & 17 & 75 & 47 & 5 & 77 & 54 & 1 & 74 \\
\hline Dyspepsia and other dis. of stomach & 33 & 27 & 69 & 47 & 7 & 75 & 60 & 2 & 67 \\
\hline Mental d.o. because of psychoactive subst. & 32 & 27 & 70 & 48 & 13 & 68 & 53 & 10 & 66 \\
\hline Pneumonia & 32 & 31 & 66 & 40 & 8 & 81 & 46 & 1 & 82 \\
\hline Dermatitis, eczema, and papulosquamous d.o. & 31 & 16 & 82 & 47 & 3 & 79 & 53 & 1 & 75 \\
\hline Other noninfective gastroenteritis and colitis & 31 & 33 & 65 & 42 & 7 & 80 & 51 & 0 & 78 \\
\hline Other dis. of the respiratory system & 29 & 25 & 75 & 46 & 8 & 75 & 56 & 2 & 71 \\
\hline Dis. of esophagus & 29 & 29 & 71 & 46 & 4 & 79 & 54 & 2 & 73 \\
\hline Other dis. of the digestive system & 29 & 27 & 73 & 48 & 7 & 74 & 58 & 2 & 69 \\
\hline Diabetes mellitus & 29 & 13 & 87 & 39 & 11 & 79 & 45 & 3 & 81 \\
\hline Glomerular and renal tubulointerstitial dis. & 28 & 27 & 74 & 46 & 6 & 77 & 51 & 2 & 76 \\
\hline Alcoholic liver disease & 28 & 29 & 72 & 44 & 21 & 64 & 50 & 17 & 62 \\
\hline Other mental and behavioral d.o. & 28 & 24 & 77 & 41 & 7 & 81 & 57 & 3 & 69 \\
\hline Paralytic ileus, intestinal obstruction excl. hemia & 27 & 28 & 74 & 43 & 8 & 78 & 55 & 2 & 72 \\
\hline HIV disease & 26 & 24 & 79 & 45 & 17 & 67 & 53 & 16 & 60 \\
\hline Other dis. of intestine & 26 & 25 & 78 & 42 & 9 & 78 & 52 & 1 & 76 \\
\hline Systemic connective tissue d.o. & 26 & 24 & 79 & 38 & 8 & 83 & 53 & 4 & 72 \\
\hline Heart failure & 25 & 30 & 74 & 41 & 9 & 79 & 48 & 8 & 73 \\
\hline Mental and behavioral d.o. because of alcohol & 25 & 47 & 57 & 36 & 25 & 68 & 40 & 17 & 72 \\
\hline Other and unspecified effects of external causes & 24 & 13 & 92 & 49 & 5 & 75 & 58 & 0 & 71 \\
\hline Dementia & 24 & 25 & 80 & 28 & 15 & 86 & 34 & 14 & 81 \\
\hline Other symptoms, signs, and abnormal findings & 23 & 24 & 82 & 39 & 3 & 87 & 46 & 0 & 83 \\
\hline Intestinal infectious dis. except diarrhea & 23 & 28 & 78 & 37 & 10 & 82 & 45 & 4 & 80 \\
\hline
\end{tabular}

Abbreviations: E, expected; excl., excluding; O, observed; R., remainder; dis., disease; d.o., disorder; subst, substance; HIV, human immunodeficiency virus.

Notes: Because of space considerations, numbers are not shown for a period length of 3 years.

Numbers are also shown for 5 and 10 years. 